\title{
LAYER PROFILES OF SOLUTIONS TO ELLIPTIC PROBLEMS UNDER PARAMETER-DEPENDENT BOUNDARY CONDITIONS
}

\author{
JORGE GARCÍA-MELIÁN, JULIO D. ROSSI AND JOSÉ C. SABINA DE LIS
}

\begin{abstract}
We consider the unique positive solution to the equation $\Delta u=u^{r}$ in $\Omega$, where $r>1$ and $\Omega$ is a smooth bounded domain of $\mathbb{R}^{N}$, under one of the boundary conditions $u=\lambda, \frac{\partial u}{\partial \nu}=\lambda, \frac{\partial u}{\partial \nu}=\lambda u$ or $\frac{\partial u}{\partial \nu}=\lambda u-u^{q}$ on $\partial \Omega, q>1$. The main interest is determining the exact layer behavior of this solution near $\partial \Omega$ in terms of the parameter $\lambda$ as $\lambda \rightarrow \infty$. Our analysis is completed with the study of the same type of problems involving the $p$-Laplacian operator.
\end{abstract}

\section{INTRODUCTION}

In this paper we are interested in determining the exact asymptotic behavior near the boundary of the unique positive solution to some reaction diffusion equations posed in smooth bounded domains $\Omega$ of $\mathbb{R}^{N}$, whose prototype is $\Delta u=u^{r}$ for $r>1$, and subject to a broad class of boundary conditions whose characteristic feature is the presence of a control parameter $\lambda$. Our analysis covers Dirichlet, Neumann and Robin conditions. In addition, a nonlinear flux condition with a logistic type growth is also studied.

To illustrate the issues we are interested in, consider first the Dirichlet problem

$$
\left\{\begin{array}{cl}
\Delta u=u^{r} & \text { in } \Omega \\
u=\lambda & \text { on } \partial \Omega
\end{array}\right.
$$

It is well known that problem (1.1) has a unique positive solution for every $\lambda>0$, which will be denoted by $u_{\lambda}$. It follows by uniqueness that $u_{\lambda}$ is increasing in $\lambda$. On one hand, it is clear that $u_{\lambda \mid \partial \Omega} \rightarrow \infty$ as $\lambda \rightarrow \infty$, while on the other hand $u_{\lambda}$ stays bounded in the interior of $\Omega$ by the unique positive solution $U$ to the boundary blow-up problem

$$
\left\{\begin{array}{cl}
\Delta u=u^{r} & \text { in } \Omega \\
u=\infty & \text { on } \partial \Omega
\end{array}\right.
$$

(cf. [10], [1], [2]). We have indeed that $u_{\lambda} \rightarrow U$ uniformly on compact sets of $\Omega$ together with its derivatives up to the second order. It is further known that the solution $U$ behaves like $A d(x)^{-\alpha}$ near $\partial \Omega$, where $d(x):=\operatorname{dist}(x, \partial \Omega)$, and

$$
\alpha=\frac{2}{r-1}, \quad A=(\alpha(\alpha+1))^{\frac{1}{r-1}} .
$$

So the following question arises: how does this singularity develop near $\partial \Omega$ as $\lambda \rightarrow \infty$ ? More precisely: which is the boundary layer behavior of $u_{\lambda}$ on $\partial \Omega$ as $\lambda$ becomes larger and larger?

It can be seen by comparison of $u_{\lambda}$ with suitable sub and supersolutions of the form $K\left(\phi+C / \lambda^{\frac{1}{\alpha}}\right)^{-\alpha}, K, C$ positive constants and $\phi$ the unique solution to $-\Delta \phi=1$ in $\Omega$ 
subject to $\phi=0$ on $\partial \Omega$, that there exist positive constants $K_{1}, K_{2}$ such that

$$
K_{1}\left(d(x)+\frac{1}{\lambda^{\frac{1}{\alpha}}}\right)^{-\alpha} \leq u_{\lambda}(x) \leq K_{2}\left(d(x)+\frac{1}{\lambda^{\frac{1}{\alpha}}}\right)^{-\alpha}
$$

in $\Omega$ for all $\lambda \geq 1$ (say). In fact and due to Hopf's principle, $\phi$ has been replaced by the distance function $d(x)$. This suggests that the behavior of the solution at a (moving) point $x$ should depend on whether $d(x)$ is of the order of $\lambda^{-\frac{1}{\alpha}}$ or not. Thus, this could be considered as a "critical scale", and it could be expected that if we approach the boundary at a larger scale, only the information furnished by the equation is relevant, while if we move with $\lambda$ at a smaller scale then only the boundary condition matters.

This is actually the situation, as our first result shows. Moreover, if the boundary is approached at the exact "boundary layer" scale $\rho_{0} \lambda^{-\frac{1}{\alpha}}$, then the asymptotic profile is affected by the coefficient $\rho_{0}$.

As customary, we will use the following notations: for $f(\lambda)$ and $g(\lambda)$ defined and positive in $(a, \infty)$ for large $a, f \gg g$ is a shorthand for $\lim _{\lambda \rightarrow \infty} f(\lambda) / g(\lambda)=\infty$. Similarly, $f \sim g$ means that $\lim _{\lambda \rightarrow \infty} f(\lambda) / g(\lambda)=l$ for a certain positive and finite $l$.

Theorem 1. [Dirichlet Conditions] Let $\alpha=\frac{2}{r-1}$ and $A=(\alpha(\alpha+1))^{\frac{1}{r-1}}$. Let $u_{\lambda}$ be the unique solution to (1.1) for $\lambda>0$. Then for every $\varepsilon>0$ there exist $\lambda_{0}>0, \delta>0, M>0$ such that

$$
(A-\varepsilon)\left(d(x)+A^{\frac{1}{\alpha}} \lambda^{-\frac{1}{\alpha}}\right)^{-\alpha}-M \leq u_{\lambda}(x) \leq(A+\varepsilon)\left(d(x)+A^{\frac{1}{\alpha}} \lambda^{-\frac{1}{\alpha}}\right)^{-\alpha}+M
$$

if $d(x) \leq \delta$ and $\lambda \geq \lambda_{0}$. In particular:

- If $\rho(\lambda)>0$ and $\rho(\lambda) \rightarrow \infty$ as $\lambda \rightarrow \infty$, then $d(x)^{\alpha} u_{\lambda}(x) \rightarrow A$ as $d(x) \rightarrow 0, \lambda \rightarrow \infty$, uniformly in $d(x) \geq \rho(\lambda) \lambda^{-\frac{1}{\alpha}}$.

- If $\rho(\lambda)>0$ and $\rho(\lambda) \rightarrow 0$ as $\lambda \rightarrow \infty$, then $\frac{u_{\lambda}(x)}{\lambda} \rightarrow 1$ as $\lambda \rightarrow \infty$, uniformly in $d(x) \leq \rho(\lambda) \lambda^{-\frac{1}{\alpha}}$.

- If $\rho_{0}>0$ and $d(x) \lambda^{\frac{1}{\alpha}} \rightarrow \rho_{0}$, then $d(x)^{\alpha} u_{\lambda}(x) \rightarrow A\left(1+A^{\frac{1}{\alpha}} \rho_{0}^{-1}\right)^{-\alpha}$ as $\lambda \rightarrow \infty$.

Similar considerations can be made for the same equation under Neumann boundary conditions, that is, for the problem

$$
\begin{cases}\Delta z=z^{r} & \text { in } \Omega \\ \frac{\partial z}{\partial \nu}=\lambda & \text { on } \partial \Omega .\end{cases}
$$

We have the following result:

Theorem 2. [Neumann Conditions] Let $\alpha=\frac{2}{r-1}$ and $A=(\alpha(\alpha+1))^{\frac{1}{r-1}}$. Consider the unique positive solution $z_{\lambda}$ to (1.4) which exists for every $\lambda>0$. Then, for every $\varepsilon>0$ there are positive values $\lambda_{0}, \delta$ and $M$ such that for $\lambda \geq \lambda_{0}$, the solution $z_{\lambda}$ is estimated in the region $0<d(x)<\delta$ as follows

$$
\begin{aligned}
(A-\varepsilon)\left(d(x)+(\alpha A)^{\frac{1}{\alpha+1}} \lambda^{-\frac{1}{\alpha+1}}\right)^{-\alpha}-M & \leq z_{\lambda}(x) \leq \\
& (A+\varepsilon)\left(d(x)+(\alpha A)^{\frac{1}{\alpha+1}} \lambda^{-\frac{1}{\alpha+1}}\right)^{-\alpha}+M .
\end{aligned}
$$

Therefore,

- $\lim _{\lambda \rightarrow \infty} d(x)^{\alpha} z_{\lambda}=A$ provided that $\delta \gg d(x) \gg \lambda^{-\frac{1}{\alpha+1}}$ uniformly as $\lambda \rightarrow \infty$,

- $\lim _{\lambda \rightarrow \infty} \lambda^{-\frac{\alpha}{\alpha+1}} z \lambda=\left(\frac{A}{\alpha^{\alpha}}\right)^{\frac{\alpha}{\alpha+1}}$ provided $d(x) \ll \lambda^{-\frac{1}{\alpha+1}}$ as $\lambda \rightarrow \infty$, 
- and finally, $\lim _{\lambda \rightarrow \infty} d(x)^{\alpha} z_{\lambda}(x) \rightarrow A\left(1+(\alpha A)^{\frac{1}{\alpha+1}} \rho_{0}^{-1}\right)^{-\alpha}$ as $\lambda \rightarrow \infty$ if $d(x) \sim$ $\rho_{0} \lambda^{-\frac{1}{\alpha+1}}$ as $\lambda \rightarrow \infty$.

The results in Theorems 1 and 2 concerning problems (1.1) and (1.4) are proved by comparing $u_{\lambda}$ and $z_{\lambda}$ with suitable sub and supersolutions in terms of $d(x)$ near the boundary, a technique which is usual when dealing with boundary blow-up problems like (1.2) (see for example [2] or [5]). With similar computations, we are also able to analyze the Robin type problem

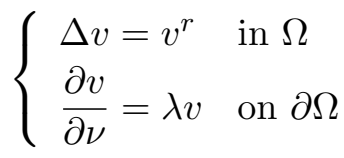

which was considered in [6] for $r>1$ (also in [7] for $0<r<1$ ). It was shown there that the positive solution $v_{\lambda}$ is unique and later proved in Theorem 7 of [9] that there exist positive constants $K_{1}, K_{2}$ such that

$$
K_{1}\left(d(x)+\frac{1}{\lambda}\right)^{-\alpha} \leq v_{\lambda}(x) \leq K_{2}\left(d(x)+\frac{1}{\lambda}\right)^{-\alpha} .
$$

in $\Omega$ if $\lambda \geq 1$. Thus similar results as for problems (1.1) and (1.4) may be expected, with a different "critical scale" of order $\lambda^{-1}$.

Theorem 3. [Robin Conditions] Let $v_{\lambda}$ be the unique solution to (1.6) for $\lambda>0$. Then for every $\varepsilon>0$ there exist $\lambda_{0}>0, \delta>0, M>0$ such that

$$
(A-\varepsilon)\left(d(x)+\frac{\alpha+\varepsilon}{\lambda}\right)^{-\alpha}-M \leq v_{\lambda}(x) \leq(A+\varepsilon)\left(d(x)+\frac{\alpha-\varepsilon}{\lambda}\right)^{-\alpha}+M
$$

if $d(x) \leq \delta, \lambda \geq \lambda_{0}$. In particular:

- If $\rho(\lambda)>0$ and $\rho(\lambda) \rightarrow \infty$ as $\lambda \rightarrow \infty$, then $d(x)^{\alpha} v_{\lambda}(x) \rightarrow A$ as $d(x) \rightarrow 0, \lambda \rightarrow \infty$, uniformly in $d(x) \geq \rho(\lambda) \lambda^{-1}$.

- If $\rho(\lambda)>0$ and $\rho(\lambda) \rightarrow 0$ as $\lambda \rightarrow \infty$, then $\frac{v_{\lambda}(x)}{\lambda^{\alpha}} \rightarrow A \alpha^{-\alpha}$ as $\lambda \rightarrow \infty$, uniformly in $d(x) \leq \rho(\lambda) \lambda^{-1}$

- If $\rho_{0}>0$ and $d(x) \lambda \rightarrow \rho_{0}$, then $d(x)^{\alpha} v_{\lambda}(x) \rightarrow A\left(1+\alpha \rho_{0}^{-1}\right)^{-\alpha}$ as $\lambda \rightarrow \infty$.

Finally, we further consider the following perturbation of problem (1.6),

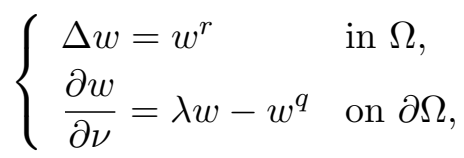

where $q>1$. Such problem can be regarded as the competition between two reactions of orders $r$ and $q$ respectively. The former is volumetric and takes place in the whole of $\Omega$ while the latter, which is represented by the term $-w^{q}$, is a surface reaction on $\partial \Omega$. Such processes are coupled with diffusion in $\Omega$ while in addition the reaction on $\partial \Omega$ is coupled to a source linear term with flux intensity $\lambda$. In other words, a logistic type flux regulates $w$ on $\partial \Omega$.

The question now is whether the presence of the surface sink term $w^{q}$ may change the behavior of solutions as $\lambda \rightarrow \infty$ with respect to that given by Theorem 3 . Indeed, observe that this problem has a unique positive solution for all $\lambda>0$, as a consequence of Theorem 1 in [8]. It will be denoted by $w_{\lambda}$. A self-contained proof of this fact and additional features of $w_{\lambda}$ are delayed to Section 2 (see the proof of Theorem 4 and Section 3 for the study of the same questions in a more general context). 
We find that two regimes are possible, depending on which of the two reaction mechanisms dominates the consumption of $u$. Such a dominance is expressed in terms of the orders $r, q$ of the reactions. When $q$ is no too big with respect to $r$, that is, $q \leq \frac{r+1}{2}$, the asymptotic behavior essentially coincides with that of problem (1.6). Indeed, the critical scale is the same, although the constants in the profile change in the borderline case $q=\frac{r+1}{2}$. Under $q \leq \frac{r+1}{2}$, the volumetric reaction $-w^{r}$ prevails over the surface reaction $-w^{q}$.

On the contrary, when $q>\frac{r+1}{2}$, solutions undergo a qualitative change in their behavior and are affected by the presence of the $q$ power. This is just the case where surface reaction is the leading degradation process. As a first approximation, it can be shown that

$$
K_{1}\left(d(x)+\frac{1}{\lambda}\right)^{-\frac{1}{q-1}} \leq w_{\lambda}(x) \leq \lambda^{\frac{1}{q-1}}
$$

in $\Omega$ for a positive constant $K_{1}$ and $\lambda \geq 1$. The leftmost inequality in (1.10) follows by constructing a subsolution while the rightmost is a consequence of Hopf's boundary lemma. These inequalities say in particular that $w_{\lambda}$ is comparable on $\partial \Omega$ with $\lambda^{\frac{1}{q-1}}$, and therefore $w_{\lambda}$ is comparable in $\Omega$, via the maximum principle, with $u_{\lambda^{\frac{1}{q-1}}}$ (the solution to (1.1) with $\lambda$ replaced by $\left.\lambda^{\frac{1}{q-1}}\right)$. Hence from (1.7) we arrive at

$$
K_{1}\left(d(x)+\frac{1}{\lambda^{\tau}}\right)^{-\alpha} \leq v_{\lambda}(x) \leq K_{2}\left(d(x)+\frac{1}{\lambda^{\tau}}\right)^{-\alpha}
$$

in $\Omega$ for $\lambda \geq 1$, where $\tau=\frac{r-1}{2(q-1)}$. This shows that the critical scale is modified and becomes of the order of $\lambda^{-\tau}$ as $\lambda \rightarrow \infty$. Hence, we obtain a similar result to those in Theorems 1, 2 and 3.

Theorem 4. Let $w_{\lambda}$ be the unique solution to (1.9) for $\lambda>0$.

A) Assume $q \leq \frac{r+1}{2}$. Then for every $\varepsilon>0$ there exist $\lambda_{0}>0, \delta>0, M>0$ such that

$$
(A-\varepsilon)\left(d(x)+\gamma_{\varepsilon}^{-} \lambda^{-\frac{1}{\alpha}}\right)^{-\alpha}-M \leq w_{\lambda}(x) \leq(A+\varepsilon)\left(d(x)+\gamma_{\varepsilon}^{+} \lambda^{-\frac{1}{\alpha}}\right)^{-\alpha}+M
$$

if $d(x) \leq \delta, \lambda \geq \lambda_{0}$, where $\gamma_{\varepsilon}^{ \pm}=\alpha \mp \varepsilon$ if $q<\frac{r+1}{2}$ and $\gamma_{\varepsilon}^{ \pm}=\alpha+A^{q-1}$ when $q=\frac{r+1}{2}$. Setting $\gamma=\left.\gamma_{\varepsilon}^{ \pm}\right|_{\varepsilon=0}$, the following properties hold:

- If $\rho(\lambda)>0$ and $\rho(\lambda) \rightarrow \infty$ as $\lambda \rightarrow \infty$, then $d(x)^{\alpha} w_{\lambda}(x) \rightarrow A$ as $d(x) \rightarrow 0, \lambda \rightarrow \infty$, uniformly in $d(x) \geq \rho(\lambda) \lambda^{-1}$.

- If $\rho(\lambda)>0$ and $\rho(\lambda) \rightarrow 0$ as $\lambda \rightarrow \infty$, then $\frac{w_{\lambda}(x)}{\lambda^{\alpha}} \rightarrow A \gamma^{-\alpha}$ as $\lambda \rightarrow \infty$, uniformly in $d(x) \leq \rho(\lambda) \lambda^{-1}$

- If $\rho_{0}>0$ and $d(x) \lambda \rightarrow \rho_{0}$, then $d(x)^{\alpha} w_{\lambda}(x) \rightarrow A\left(1+\gamma \rho_{0}^{-1}\right)^{-\alpha}$ as $\lambda \rightarrow \infty$.

B) Suppose, on the contrary, $q>\frac{r+1}{2}$. Then the solution $w_{\lambda}$ verifies

$$
(A-\varepsilon)\left(d(x)+A^{\frac{1}{\alpha}} \lambda^{-\tau}\right)^{-\alpha}-M \leq w_{\lambda}(x) \leq(A+\varepsilon)\left(d(x)+A^{\frac{1}{\alpha}} \lambda^{-\tau}\right)^{-\alpha}+M
$$

if $d(x) \leq \delta, \lambda \geq \lambda_{0}$. Hence:

- If $\rho(\lambda)>0$ and $\rho(\lambda) \rightarrow \infty$ as $\lambda \rightarrow \infty$, then $d(x)^{\alpha} w_{\lambda}(x) \rightarrow A$ as $d(x) \rightarrow 0, \lambda \rightarrow \infty$, uniformly in $d(x) \geq \rho(\lambda) \lambda^{-\tau}$.

- If $\rho(\lambda)>0$ and $\rho(\lambda) \rightarrow 0$ as $\lambda \rightarrow \infty$, then $\frac{w_{\lambda}(x)}{\lambda^{\frac{1}{q-1}}} \rightarrow 1$ as $\lambda \rightarrow \infty$, uniformly in $d(x) \leq \rho(\lambda) \lambda^{-\tau}$.

- If $\rho_{0}>0$ and $d(x) \lambda^{\tau} \rightarrow \rho_{0}$, then $d(x)^{\alpha} w_{\lambda}(x) \rightarrow A\left(1+A^{\frac{1}{\alpha}} \rho_{0}^{-1}\right)^{-\alpha}$ as $\lambda \rightarrow \infty$. 
Finally, we also consider briefly the nonlinear diffusion version of the problems which have been studied before, namely

$$
\begin{cases}\Delta_{p} u=u^{r} & x \in \Omega \\ \mathcal{B}_{p}(u, \lambda)=0 & x \in \partial \Omega,\end{cases}
$$

where $\Delta_{p}$ stands for the $p$-Laplacian, which is weakly defined in $W^{1, p}(\Omega)$ as $\Delta_{p} u=$ $\operatorname{div}\left(|\nabla u|^{p-2} \nabla u\right), p>1, r>p-1$ and $\mathcal{B}_{p}(u, \lambda)$ stands for the natural generalization of each of the previous boundary conditions. See Section 3 for the statement of the corresponding results.

The rest of the paper is organized as follows: in Section 2 we prove Theorems 1, 2, 3 and 4 , while the analysis of the nonlinear diffusion problem (1.13) is developed in Section 3.

\section{LINEAR DIFFUSION}

In this section we prove Theorems 1, 2, 3 and 4. In all cases we look at the unique solution $u$ to the problem under consideration, i.e. (1.1), (1.4), (1.6) and (1.9), respectively, as the unique solution $\psi$ to an auxiliary problem near the boundary. Namely,

$$
\left\{\begin{array}{cl}
\Delta \psi=\psi^{r} & 0<d(x)<\delta, \\
\psi=u & d(x)=\delta, \\
\mathcal{B}(\psi, \lambda)=0 & x \in \partial \Omega,
\end{array}\right.
$$

for $\delta>0$ small, where $\mathcal{B}=\mathcal{B}(\psi, \lambda)$ stands for the corresponding boundary operator in each case, i. e. $\mathcal{B}(\psi, \lambda)=\psi-\lambda, \mathcal{B}(\psi, \lambda)=\frac{\partial \psi}{\partial \nu}-\lambda, \mathcal{B}(\psi, \lambda)=\frac{\partial \psi}{\partial \nu}-\lambda \psi$ and $\mathcal{B}(\psi, \lambda)=\frac{\partial \psi}{\partial \nu}-\lambda \psi+\psi^{q}$, respectively. Then, we are looking for appropriate sub and supersolutions to (2.14) to obtain the desired estimates.

Proof of Theorem 1. We begin with the Dirichlet problem (1.1) which, as already mentioned, has a unique solution $u_{\lambda}$ for every $\lambda>0$ which is increasing in $\lambda$ and stays finite in $\Omega$ as $\lambda \rightarrow \infty$ ([1], [5]). Recall in addition that since $\Omega$ is $C^{2}$, there exists $\delta_{0}>0$ such that $d$ is $C^{2}$ in $d(x)<\delta_{0}$, with $|\nabla d|=1$ there. To find a suitable supersolution choose $\varepsilon>0$ and let

$$
\bar{u}(x)=(A+\varepsilon)\left(d(x)+A^{\frac{1}{\alpha}} \lambda^{-\frac{1}{\alpha}}\right)^{-\alpha}+M
$$

for $M>0$. We claim that $\bar{u}$ is a supersolution to (1.1) if $d<\delta$ and $\lambda \geq \lambda_{0}$, where $\delta<\delta_{0}$ is small and $\lambda_{0}$ is large (both depending on $\varepsilon$ ). This will hold provided

$$
\begin{aligned}
\alpha(\alpha+1)(A+\varepsilon)\left(d(x)+A^{\frac{1}{\alpha}} \lambda^{-\frac{1}{\alpha}}\right)^{-\alpha-2}-\alpha(A+\varepsilon) & \left(d(x)+A^{\frac{1}{\alpha}} \lambda^{-\frac{1}{\alpha}}\right)^{-\alpha-1} \Delta d \\
\leq & \left((A+\varepsilon)\left(d(x)+A^{\frac{1}{\alpha}} \lambda^{-\frac{1}{\alpha}}\right)^{-\alpha}+M\right)^{r}
\end{aligned}
$$

in $d(x)<\delta$. In particular, it is enough to have

$$
\alpha(\alpha+1)-\alpha\left(d(x)+A^{\frac{1}{\alpha}} \lambda^{-\frac{1}{\alpha}}\right) \Delta d \leq(A+\varepsilon)^{r-1}
$$

in $d(x)<\delta$, which is always possible if $\lambda$ is large enough and $\delta$ small enough, since $\alpha(\alpha+1)=A^{r-1}$. On the other hand, notice that $u=\lambda$ on $\partial \Omega$. Since $M$ can be selected so that $M \geq u_{\lambda}$ on $d(x)=\delta$ for large $\lambda$ (recall that $u_{\lambda}$ stays finite on $d=\delta$ as $\lambda \rightarrow \infty$ ), we obtain by comparison that $u_{\lambda} \leq \bar{u}$ in $d(x)<\delta$. 
It is analogously shown that $\underline{u}=(A-\varepsilon)\left(d(x)+A^{\frac{1}{\alpha}} \lambda^{-\frac{1}{\alpha}}\right)^{-\alpha}-M$ is a subsolution in $d(x)<\delta$ with $\underline{u}=\lambda$ on $\partial \Omega$ and $\underline{u} \leq u_{\lambda}$ on $d(x)=\delta$ if $M$ is chosen appropriately. Thus $\underline{u} \leq u_{\lambda}$ in $d(x)<\delta$ and (1.3) is proved.

To conclude the proof, notice that if $x \in \Omega$ (depending on $\lambda$ ) is such that $d(x) \rightarrow 0$ and $d(x) \lambda^{\frac{1}{\alpha}} \rightarrow \infty$ as $\lambda \rightarrow \infty$ then according to (1.3) we have for every $\varepsilon>0$

$$
d(x)^{\alpha} u_{\lambda}(x) \leq(A+\varepsilon)\left(1+A^{\frac{1}{\alpha}}\left(d(x) \lambda^{\frac{1}{\alpha}}\right)^{-1}\right)^{-\alpha}+M d(x)^{\alpha}
$$

and thus $\lim \sup d(x)^{\alpha} u_{\lambda}(x) \leq A$. The lower inequality follows similarly, as well as the remaining assertions of the theorem.

Proof of Theorem 2. Let us consider now problem (1.4). Its main features are next described for the sake of completeness (see also Section 3). First, (1.4) can only admit a unique positive solution for $\lambda>0$. To get existence, a supersolution (whose structure is suitable for our purposes here) is provided by

$$
\bar{z}=B(\phi+\mu)^{-\alpha},
$$

with $\alpha=2 /(r-1), \mu=\left(-\alpha\left\{\min _{\partial \Omega} \frac{\partial \phi}{\partial \nu}\right\} B / \lambda\right)^{\frac{1}{\alpha+1}}$ and $\phi(x)$ the function introduced in Section 1 , and where

$$
B_{+}=\left(\phi_{\max }+\alpha \mu\right)^{\frac{1}{r-1}},
$$

$\phi_{\max }=\max _{\Omega}\left\{\alpha(\alpha+1)|\nabla \phi|^{2}+\alpha \phi\right\}$. A subsolution is similarly found in the form

$$
\underline{z}=B(\phi+\mu)^{-\alpha},
$$

where now $\mu=\left(-\alpha\left\{\max _{\partial \Omega} \frac{\partial \phi}{\partial \nu}\right\} B / \lambda\right)^{\frac{1}{\alpha+1}}$ and

$$
B_{-}=\left(\phi_{\min }+\alpha \mu\right)^{\frac{1}{r-1}},
$$

with $\phi_{\min }=\min _{\Omega}\left\{\alpha(\alpha+1)|\nabla \phi|^{2}+\alpha \phi\right\}$.

Since $\theta \underline{z}$ is, for small $0<\theta<1$, a subsolution smaller than $\bar{z}$ we find a unique solution $z_{\lambda}$ satisfying $\theta \underline{z} \leq z_{\lambda} \leq \bar{z}$. The same reasoning allows us to show that the solution $z_{\lambda}$ satisfies $\underline{z} \leq z_{\lambda} \leq \bar{z}$ for all $\lambda>0$ (notice that both $\underline{z}, \bar{z}$ depend on $\lambda$ ).

On the other hand observe that $B_{ \pm} \rightarrow \infty$ as $\lambda \rightarrow 0$ while $B_{ \pm} \rightarrow B_{ \pm}(\infty)$ as $\lambda \rightarrow \infty$, where $B_{ \pm}(\infty)$ are the values obtained by solving the equations for $B_{ \pm}$when setting $\mu=0$. Two conclusions can be obtained from this fact. The first one is that $z_{\lambda}$ bifurcates from zero as $\lambda \rightarrow 0$ while the second one is that $z_{\lambda}$, which is increasing in $\lambda$, keeps finite in $\Omega$ as $\lambda \rightarrow \infty$, with limit $z_{\infty}=\lim _{\lambda \rightarrow \infty} z_{\lambda}$ satisfying

$$
\frac{B_{-}(\infty)}{\phi^{\alpha}} \leq z_{\infty} \leq \frac{B_{+}(\infty)}{\phi^{\alpha}},
$$

in $\Omega$. Furthermore, by standard $L^{p}$ estimates and bootstrapping it follows that $z_{\infty}$ coincides with $U$, the solution to the blow-up problem (1.2).

We can now proceed to prove estimate (1.5). By arguing as before one finds that for every $\varepsilon>0$ there exist positive $M$ and $\delta$ such that

$$
\bar{z}_{\lambda}:=(A+\varepsilon)\left(d+(\alpha A)^{\frac{1}{\alpha+1}} \lambda^{-\frac{1}{\alpha+1}}\right)^{-\alpha}+M,
$$

defines a subsolution to problem (2.14) while

$$
\underline{z}_{\lambda}:=(A-\varepsilon)\left(d+(\alpha A)^{\frac{1}{\alpha+1}} \lambda^{-\frac{1}{\alpha+1}}\right)^{-\alpha}-M,
$$


constitutes a comparable subsolution. Therefore, the solution to (1.4) satisfies

$$
(A-\varepsilon)\left(d+(\alpha A)^{\frac{1}{\alpha+1}} \lambda^{-\frac{1}{\alpha+1}}\right)^{-\alpha}-M \leq z_{\lambda} \leq(A+\varepsilon)\left(d+(\alpha A)^{\frac{1}{\alpha+1}} \lambda^{-\frac{1}{\alpha+1}}\right)^{-\alpha}+M
$$

in $0<d(x)<\delta$. From this inequality, which is just (1.5), the remaining assertions follow.

Proof of Theorem 3. First recall that the features on existence, uniqueness and increasing character of a positive solution $v_{\lambda}$ to (1.6), together with its finiteness as $\lambda \rightarrow \infty$ are contained in [6]. Next, for small $\varepsilon>0$ we look for a supersolution to (2.14) of the form:

$$
\bar{v}=(A+\varepsilon)(d+\mu)^{\alpha}+M,
$$

with a small $\delta>0$ and having in mind that $\mu \rightarrow 0$ as $\lambda \rightarrow \infty$. Setting $\eta=(A+\varepsilon)^{r-1}-A^{r-1}$, $\delta$ is chosen so that

$$
\sup _{0<d<\delta} \alpha|\Delta d|<\frac{\eta}{2}
$$

This gives a supersolution to the equation provided that

$$
\sup _{0<d<\delta} \mu|\Delta d|<\frac{\eta}{2}
$$

Regarding the boundary condition one needs,

$$
\alpha \mu^{-1} \geq \lambda\left(1+\frac{M}{A+\varepsilon} \mu^{\alpha}\right)
$$

for a small $\mu$, where $M$ is considered a parameter. Then we set $\mu=\frac{\alpha-\varepsilon}{\lambda}$ and (2.16) holds for large $\lambda$ (depending on $\varepsilon$ and $M$ ). Now, $M$ is chosen such that

$$
v_{\lambda} \leq(A+\varepsilon)\left(d+\frac{\alpha-\varepsilon}{\lambda}\right)+M
$$

on $d(x)=\delta$ for $\lambda$ greater than a certain amount. Finally, its size is also chosen so that (2.15) is satisfied. In this way the construction of the supersolution $\bar{v}$ is accomplished and we have $v_{\lambda} \leq \bar{v}$.

The subsolution is given by $\underline{v}=(A-\varepsilon)\left(d(x)+\frac{\alpha+\varepsilon}{\lambda}\right)^{-\alpha}-M$, and a similar argument gives (1.8). The remaining assertions of the theorem easily follow.

Proof of Theorem 4. We are postponing the detailed discussion of the features of problem (1.9) to the end of the proof. So, let us begin with the case $q<\frac{r+1}{2}$ whose analysis follows the general lines of Theorem 3. We seek a supersolution to (2.14) in the form,

$$
\bar{w}=(A+\varepsilon)(d(x)+\mu)^{-\alpha}+M,
$$

for $\delta>0$ small. Then the computations run as in Theorem 3 but, in order to adjust the boundary condition, equation (2.16) reads now

$$
\alpha \mu^{-\alpha-1}+(A+\varepsilon)^{q-1} \mu^{-\alpha q} \geq \lambda \mu^{-\alpha}\left(1+\frac{M}{A+\varepsilon} \mu^{\alpha}\right)
$$

where $\mu$ is going to vanish as $\lambda \rightarrow \infty$. Now $\alpha+1>\alpha q$ if $q<\frac{r+1}{2}$ thus (2.17) is better expressed as

$$
\left(\alpha+(A+\varepsilon)^{q-1} \mu^{\alpha+1-\alpha q}\right) \mu^{-1} \geq \lambda\left(1+\frac{M}{A+\varepsilon} \mu^{\alpha}\right)
$$


and choosing $\mu=\frac{\alpha-\varepsilon}{\lambda}$ then (2.18) holds if $\lambda$ is large (depending on $\varepsilon$ and $M$ ). The remaining details leading to estimate (1.11) -particularly the production of a corresponding subsolution to (2.14)- coincide with those in Theorem 3.

In the case $q=\frac{r+1}{2}$, both the supersolution and the subsolution need to be slightly modified. In fact, (2.18) becomes

$$
\left(\alpha+(A+\varepsilon)^{q-1}\right) \mu^{-1} \geq \lambda\left(1+\frac{M}{A+\varepsilon} \mu^{\alpha}\right),
$$

and the suitable supersolution is given by

$$
\bar{w}=(A+\varepsilon)\left(d(x)+\frac{\alpha+A^{q-1}}{\lambda}\right)^{-\alpha}+M,
$$

being the corresponding subsolution accordingly corrected. We leave the details to the reader.

Hence it only remains to consider the case $q>\frac{r+1}{2}$. In this range $\alpha q>\alpha+1$ and $(2.17)$ should be better written as

$$
\left((A+\varepsilon)^{q-1}+\alpha \mu^{\alpha q-\alpha-1}\right) \mu^{-\alpha q} \geq \lambda \mu^{-\alpha}\left(1+\frac{M}{A+\varepsilon} \mu^{\alpha}\right) .
$$

Thus (2.19) is satisfied for $\lambda$ large provided that

$$
\mu=\left(\frac{A^{q-1}}{\lambda}\right)^{\frac{1}{\alpha(q-1)}}=\frac{A^{\frac{1}{\alpha}}}{\lambda^{\tau}},
$$

with $\tau=\frac{r-1}{2(q-1)}$. By repeating the steps in Theorem 3 we conclude that

$$
\bar{w}=(A+\varepsilon)\left(d(x)+\frac{A^{\frac{1}{\alpha}}}{\lambda^{\tau}}\right)^{-\alpha}+M
$$

defines a supersolution to (2.14) for a suitable choice of $M$ (which depends on that of $\varepsilon$ ). Similarly, a comparable subsolution is provided by

$$
\underline{w}=(A-\varepsilon)\left(d(x)+\frac{A^{\frac{1}{\alpha}}}{\lambda^{\tau}}\right)^{-\alpha}+M
$$

and the proof of estimate (1.12) in part B) is completed.

To finish the proof, let us include a self contained account on problem (1.9) (see also Section 3). We are only borrowing from [8] the uniqueness of a positive solution. To get existence observe that the solution $v_{\lambda}$ to (1.6) provide us with a supersolution. A subsolution is produced by using once again the ansatz

$$
\underline{w}=B(\phi+\mu)^{-\alpha} \text {. }
$$

To get $\Delta w \geq w^{r}$ in $\Omega$ it suffices with

$$
B^{r-1}-\alpha \mu \leq \phi_{\min },
$$

where $\phi_{\min }=\min _{\Omega}\left\{\alpha(\alpha+1)|\nabla \phi|^{2}+\alpha \phi\right\}$. The corresponding inequality with the boundary condition is achieved if

$$
\lambda \geq \frac{\alpha(-\partial \phi / \partial \nu)_{\max }+B^{q-1} \mu^{1-\alpha(q-1)}}{\mu}
$$


when $q \leq \frac{r+1}{2}$ or

$$
\lambda \geq \frac{B^{q-1}+\alpha(-\partial \phi / \partial \nu)_{\max } \mu^{\alpha(q-1)-1}}{\mu^{\alpha(q-1)}}
$$

for $q>\frac{r+1}{2}$. The subsolution $\underline{w}$ is obtained in the case $q \leq \frac{r+1}{2}$ by taking equalities in both (2.20) and (2.21), setting $\mu=\frac{B^{q-1}-\phi_{\min }}{\alpha}$ and substituting in (2.21) to get a decreasing smooth function $B=B(\lambda)$ such that $B \rightarrow \infty$ as $\lambda \rightarrow 0$ while $B \rightarrow B(\infty):=\phi_{\min }^{r-1}$ as $\lambda \rightarrow \infty$. Thus, such subsolution $\underline{w}$ satisfies

$$
\lim _{\lambda \rightarrow 0} \underline{w}=0,
$$

uniformly in $\Omega$ while $\underline{w} \rightarrow B(\infty) \phi^{-\alpha}$ uniformly on compacts of $\Omega$ as $\lambda \rightarrow \infty$. A subsolution $\underline{w}$ with entirely the same properties is also constructed in this way when $q>\frac{r+1}{2}$. Finally and using the uniqueness together with the fact that $\theta \underline{w}$ and $M v_{\lambda}$ define a sub and a supersolution provided $0<\theta \leq 1$ and $M \geq 1$, respectively, we find a solution $w_{\lambda}$ to (1.9) satisfying

$$
\underline{w} \leq w_{\lambda} \leq v_{\lambda} .
$$

This gives both the bifurcation of $w_{\lambda}$ from zero at $\lambda=0$ and its finiteness as $\lambda \rightarrow \infty$. The increasing, and indeed, continuous character of $w_{\lambda}$ with respect $\lambda$ is a consequence of the fact that $w_{\lambda_{1}}$ defines a supersolution to (1.9) if $\lambda_{1} \geq \lambda$. Finally, and just as in the previous problems $\lim _{\lambda \rightarrow \infty} w_{\lambda}=U$ in $C^{2}(\Omega)$ where $U$ is the solution to (1.2).

\section{NONLINEAR DIFFUSION}

In the present section we are extending the previous results to the framework of nonlinear diffusion. Specifically, we are dealing with the class of problems (1.13)

$$
\begin{cases}\Delta_{p} u=u^{r} & x \in \Omega \\ \mathcal{B}_{p}(u, \lambda)=0 & x \in \partial \Omega,\end{cases}
$$

where $\Delta_{p}, p>1$, stands for the $p$-Laplacian operator. It will be assumed that $r>p-1$ while the boundary conditions cover the natural extensions of all of those considered in the previous sections. Namely,

$$
\begin{aligned}
& \mathcal{B}_{p}(u, \lambda)=u-\lambda \quad \text { (Dirichlet), } \\
& \mathcal{B}_{p}(u, \lambda)=|\nabla u|^{p-2} \frac{\partial u}{\partial \nu}-\lambda \quad \text { (Neumann), } \\
& \mathcal{B}_{p}(u, \lambda)=|\nabla u|^{p-2} \frac{\partial u}{\partial \nu}-\lambda u^{p-1} \quad \text { (Robin-type) }
\end{aligned}
$$

and the nonlinear flux condition under logistic growth

$$
\mathcal{B}_{p}(u, \lambda)=|\nabla u|^{p-2} \frac{\partial u}{\partial \nu}-\lambda u^{p-1}+u^{q}
$$

with $q>p-1$. In all cases, $\lambda$ will be considered as a positive parameter. We are labeling the problem (1.13) under those boundary conditions as (D), (N), (R) and (L) respectively.

That problem (1.13) admits, when subject to Dirichlet conditions, a unique weak positive solution $u_{\lambda} \in W^{1, p}(\Omega) \cap L^{\infty}(\Omega)$ which increases with $\lambda \in(0, \infty)$, bifurcates from zero at $\lambda=0$ and keeps finite in $\Omega$ as $\lambda \rightarrow \infty$, are all essentially well-known facts. Moreover, $\lim _{\lambda \rightarrow \infty} u_{\lambda}=U_{p}$ where $u=U_{p}$ is the unique solution to the problem

$$
\left\{\begin{array}{cl}
\Delta_{p} u=u^{r} & \text { in } \Omega \\
u=\infty & \text { on } \partial \Omega .
\end{array}\right.
$$


See [4] and [13] where the existence and uniqueness of a solution to (3.23) is studied.

However, the properties of existence, uniqueness, behavior in $\lambda$-specially the limit values at $\lambda=0, \infty$ - for the remaining cases of the boundary value problem (1.13), i.e. $(\mathrm{N}),(\mathrm{R})$ and $(\mathrm{L})$, need to be properly stated. Being our final goal the study of the boundary layer formation for all those problems, we are next giving a self contained account on the former properties, leaving the layer analysis for a final subsection. Thus we proceed to deal with all these questions in turn.

3.1. Uniqueness. The uniqueness of a positive weak solution to $(\mathrm{N})$ is standard. However, this is not the case for problems $(\mathrm{N})$ and $(\mathrm{L})$. In both cases, the proof in Lemma 13 of [8] can be adapted to the the $p$-Laplacian framework by employing the approach in Lemma 3.1 of [12].

3.2. Existence. A first useful remark is that provided $\underline{u}, \bar{u} \in W^{1, p}(\Omega) \cap L^{\infty}(\Omega)$ is a sub and supersolution pair to (1.13) with either of the boundary conditions listed above, then $\theta \underline{u}, M \bar{u}$ is a new such pair whenever $0<\theta \leq 1 \leq M$. This allows us to produce comparable sub and supersolutions by starting on any arbitrary pair $\underline{u}, \bar{u}$. On the other hand, for its immediate use in the constructions that follow it is convenient to introduce the function $\phi_{p} \in W^{1, p}(\Omega) \cap L^{\infty}(\Omega)$ which is the unique weak solution to

$$
\left\{\begin{array}{cl}
-\Delta_{p} u=1 & x \in \Omega, \\
u=0 & x \in \partial \Omega .
\end{array}\right.
$$

It can be shown that $\phi_{p} \in C^{1, \gamma}(\bar{\Omega})$ for a certain $0<\gamma<1$ ([3], [11], [14]). Moreover, due to the strong maximum principle $([15])$ it follows that $\phi_{p}>0$ together with $\nabla \phi_{p} \neq 0$ for all $x \in \bar{\Omega}$ such that $d(x)=\operatorname{dist}(x, \partial \Omega) \leq \delta$ (for a small $\delta>0)$.

Thus, it is possible to construct weak positive sub and supersolutions $\underline{w}, \bar{w} \in W^{1, p}(\Omega) \cap$ $L^{\infty}(\Omega)$ to $(1.13)$ (under all boundary conditions) in the form

$$
\underline{w}=B_{-}\left(\phi_{p}+\mu_{-}\right)^{-\beta} \quad \bar{w}=B_{+}\left(\phi_{p}+\mu_{+}\right)^{-\beta},
$$

with

$$
\beta=\frac{p}{r-p+1},
$$

where $\mu_{ \pm}, B_{ \pm}$are decreasing functions of $\lambda \in(0, \infty)$ which depend on the boundary conditions. They satisfy

$$
\lim _{\lambda \rightarrow 0} \mu_{ \pm}=\infty \quad \lim _{\lambda \rightarrow \infty} \mu_{ \pm}=0
$$

and

$$
\lim _{\lambda \rightarrow 0} B_{ \pm}=\infty \quad \lim _{\lambda \rightarrow \infty} B_{ \pm}=B_{p, \pm}(\infty)
$$

being

$$
B_{p,-}(\infty)=\left\{\phi_{p, \min }\right\}^{\frac{1}{r-p+1}} \quad B_{p,+}(\infty)=\left\{\phi_{p, \max }\right\}^{\frac{1}{r-p+1}}
$$

and where

$$
\phi_{p, \min }=\min _{\Omega}\left\{(p-1) \beta^{p-1}(\beta+1)\left|\nabla \phi_{p}\right|^{p}+\beta^{p-1} \phi_{p}\right\}
$$

while

$$
\phi_{p, \max }=\max _{\Omega}\left\{(p-1) \beta^{p-1}(\beta+1)\left|\nabla \phi_{p}\right|^{p}+\beta^{p-1} \phi_{p}\right\} .
$$


Furthermore, the functions $B$ and $\mu$ are related through the expressions

$$
\begin{aligned}
& B_{-}^{r-p+1}-\beta^{p-1} \mu=\phi_{p, \text { min }} \\
& B_{+}^{r-p+1}-\beta^{p-1} \mu=\phi_{p, \text { max }} .
\end{aligned}
$$

A detailed proof of previous assertions is omitted for brevity. On the other hand, it is important to point out that when checking that $\underline{w}$ and $\bar{w}$ are a sub and a supersolution, computations must be necessarily performed in a weak sense since, in general, $\phi_{p}$ does not have two classical derivatives.

Therefore, and by employing the previous remark, it is possible to find a unique positive weak solution $u_{\lambda} \in W^{1, p}(\Omega) \cap L^{\infty}(\Omega)$ to problem (1.13) with either of the boundary conditions $(\mathrm{D}),(\mathrm{N}),(\mathrm{R})$ and $(\mathrm{L})$, and this solution satisfies the inequalities

$$
\underline{w} \leq u_{\lambda} \leq \bar{w} .
$$

Moreover, in view of the results in [11], we have $u_{\lambda} \in C^{1, \gamma}(\bar{\Omega})$ for a certain $0<\gamma<1$.

3.3. Limit behavior as $\lambda \rightarrow 0, \lambda \rightarrow \infty$. That the solution $u_{\lambda}$ to (1.13) obtained in the preceding paragraph increases in $\lambda \in(0, \infty)$, follows from uniqueness and the fact that $u_{\lambda}$ constitutes a subsolution to problem $(1.13)_{\lambda^{\prime}}$ whenever $\lambda^{\prime} \geq \lambda$. On the other hand, relations (3.27) together with (3.25) and (3.26) imply that both $\underline{w} \rightarrow 0, \bar{w} \rightarrow 0$ uniformly in $\Omega$ as $\lambda \rightarrow 0$. In virtue of (3.28) the same happens to $u_{\lambda}$ and it bifurcates from zero at $\lambda=0$.

As for the finiteness of $u_{\lambda}$ as $\lambda \rightarrow \infty$ we can conclude from (3.28) and the expression for $\underline{w}, \bar{w}$ and (3.26) that the limit

$$
u_{\infty}=\lim _{\lambda \rightarrow \infty} u_{\lambda}
$$

holds uniformly on compacts of $\Omega$. Furthermore, in view of the $C^{1, \gamma}$ estimates in [11] it also holds in $C^{1, \gamma}(\Omega)$ for a certain $\gamma \in(0,1)$. Moreover,

$$
\frac{B_{p,-}(\infty)}{\phi_{p}^{\beta}} \leq u_{\infty} \leq \frac{B_{p,+}(\infty)}{\phi_{p}^{\beta}} .
$$

Since this implies that $\lim _{d(x) \rightarrow 0} u_{\infty}=\infty$ then $u_{\infty}$ defines a positive weak solution to (3.23) and thus it coincides with $U_{p}$.

3.4. Boundary layer behavior as $\lambda \rightarrow \infty$. Once the existence and uniqueness of a positive solution $u_{\lambda}$ to (1.13) together with its finiteness as $\lambda \rightarrow \infty$ have been settled down for all boundary conditions $(\mathrm{D}),(\mathrm{N}),(\mathrm{R})$, and $(\mathrm{L})$, the boundary layer behavior of $u_{\lambda}$ near $\partial \Omega$ can be analyzed.

We are next stating the extensions of the results in Sections 1, 2 to the case of the $p$ Laplacian. As in Section 2, such results are obtained by introducing the natural extension of the auxiliary boundary value problem (2.14). Namely,

$$
\left\{\begin{array}{cl}
\Delta_{p} u=u^{r} & 0<d(x)<\delta, \\
u=u_{\lambda} & d(x)=\delta, \\
\mathcal{B}_{p}(u, \lambda)=0 & x \in \partial \Omega,
\end{array}\right.
$$

which exhibits $u=u_{\lambda}$ as its unique positive solution. Thus one proceeds to produce a suitable pair of sub and supersolution $\underline{u}, \bar{u}$ for each one of the boundary conditions. Such sub and supersolutions have the form

$$
\underline{u}=\left(A_{p}-\varepsilon\right)\left(d+\mu_{-}\right)^{-\beta} \quad \bar{u}=\left(A_{p}+\varepsilon\right)\left(d+\mu_{+}\right)^{-\beta},
$$


where $A=A_{p}$ satisfies $A^{q-p+1}=(p-1) \beta^{p-1}(\beta+1)$ and $\mu_{ \pm}=\mu_{ \pm}(\lambda)$ are appropriate functions of $\lambda$ which depend on the boundary conditions and which are explicitly given below. Computations, which are entirely similar to the ones in Section 2, are going to be omitted for brevity. The boundary layer features corresponding to each problem are now listed in turn. Recall that $\beta=\frac{p}{r-p+1}$, and $A_{p}$ is given by $A_{p}^{q-p+1}=(p-1) \beta^{p-1}(\beta+1)$.

Dirichlet problem. For every $\varepsilon>0$, there exist positive $\delta=\delta(\varepsilon), \lambda(\varepsilon)$ and $M=M(\varepsilon)$ such that the solution $u_{\lambda}$ to $(\mathrm{D})$ satisfies

$$
\frac{A_{p}-\varepsilon}{\left(d(x)+A_{p}^{\frac{1}{\beta}} \lambda^{-\frac{1}{\beta}}\right)^{\beta}}-M \leq u_{\lambda} \leq \frac{A_{p}+\varepsilon}{\left(d(x)+A_{p}^{\frac{1}{\beta}} \lambda^{-\frac{1}{\beta}}\right)^{\beta}}+M,
$$

for $d(x)<\delta$ and $\lambda \geq \lambda(\varepsilon)$. Then, the critical scale is $\lambda^{-\frac{1}{\beta}}$ and

$$
u_{\lambda} \sim \frac{A_{p}}{d(x)^{\beta}},
$$

for $1 \gg d(x) \gg \lambda^{-\frac{1}{\beta}}$ as $\lambda \rightarrow \infty$. In addition,

$$
u_{\lambda} \sim \lambda
$$

provided $d(x) \ll \lambda^{-\frac{1}{\beta}}$ as $\lambda \rightarrow \infty$.

Neumann problem. The positive solution $u_{\lambda}$ to $(\mathrm{N})$ satisfies the estimates

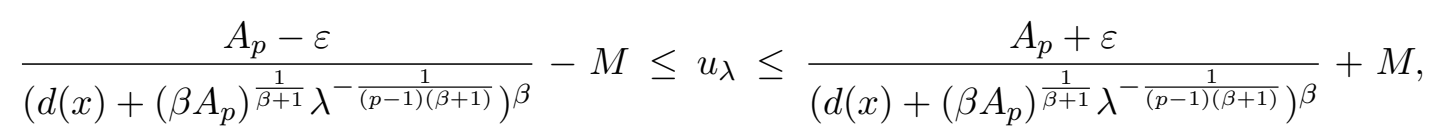

for every prefixed $\varepsilon>0$, provided that $d(x)<\delta \leq \delta(\varepsilon), \lambda \geq \lambda(\varepsilon)$ and $M=M(\varepsilon)$. That is why the critical scale becomes $\lambda^{-\frac{1}{(p-1)(\beta+1)}}$ and the inner approximation (3.30) holds provided $1 \gg d(x) \gg \lambda^{-\frac{1}{(p-1)(\beta+1)}}$ as $\lambda \rightarrow \infty$. While,

$$
u_{\lambda} \sim\left(\frac{A_{p}}{\beta^{\beta}}\right)^{\frac{1}{\beta+1}} \lambda^{\frac{\beta}{(p-1)(\beta+1)}}
$$

whenever $d(x) \ll \lambda^{-\frac{1}{(p-1)(\beta+1)}}$ as $\lambda \rightarrow \infty$.

Robin-type problem. For each $\varepsilon>0$, the positive solution $u_{\lambda}$ to $(\mathrm{R})$ satisfies the estimates

$$
\frac{A_{p}-\varepsilon}{\left(d(x)+(\beta+\varepsilon) \lambda^{-\frac{1}{p-1}}\right)^{\beta}}-M \leq u_{\lambda} \leq \frac{A_{p}+\varepsilon}{\left(d(x)+(\beta-\varepsilon) \lambda^{-\frac{1}{p-1}}\right)^{\beta}}+M,
$$

if $x$ lies in the strip $0<d(x)<\delta, M=M(\varepsilon)$ and $\lambda$ becomes arbitrarily large. The critical scale becomes $\lambda^{-\frac{1}{p-1}}$ and the inner estimate (3.30) holds if $1 \gg d(x) \gg \lambda^{-\frac{1}{p-1}}$ as $\lambda \rightarrow \infty$. In addition,

$$
u_{\lambda} \sim \frac{A_{p}}{\beta^{\beta}} \lambda^{\frac{\beta}{p-1}}
$$

provided that $d(x) \ll \lambda^{-\frac{1}{p-1}}$ as $\lambda \rightarrow \infty$.

Nonlinear flux problem: logistic growth. Just as in the linear diffusion case (1.9), problem (L) exhibits two regimes: A) $q \leq \frac{r+1}{p^{\prime}}, p^{\prime}=\frac{p}{p-1}$, corresponding to volumetric reaction dominance and B) $q>\frac{r+1}{p^{\prime}}$, which means the prevalence of the surface reaction. 
In case A) the boundary estimates and layer behavior of the solution $u_{\lambda}$ to $(\mathrm{L})$ becomes exactly the same as in the case of the Robin-type problem if $q<\frac{r+1}{p^{\prime}}$ (compare with Theorem 4, A)). When $q=\frac{r+1}{p^{\prime}}$, estimate (3.31) becomes

$$
\begin{aligned}
\frac{A_{p}-\varepsilon}{\left(d(x)+\left(\beta^{p-1}+A_{p}^{q-p+1}\right)^{\frac{1}{p-1}} \lambda^{-\frac{1}{p-1}}\right)^{\beta}}-M & \leq u_{\lambda} \leq \\
& \frac{A_{p}+\varepsilon}{\left(d(x)+\left(\beta^{p-1}+A_{p}^{q-p+1}\right)^{\frac{1}{p-1}} \lambda^{-\frac{1}{p-1}}\right)^{\beta}}+M,
\end{aligned}
$$

for every $\varepsilon>0$ when $0<d(x)<\delta(\varepsilon), M=M(\varepsilon)$ and $\lambda$ is large. The critical scale and inner behavior (3.30) remain the same as in the Robin-type problem (R) while the outer estimate becomes

$$
u_{\lambda} \sim \frac{A_{p}}{\left(\beta^{p-1}+A_{p}^{q-p+1}\right)^{\frac{\beta}{p-1}}} \lambda^{\frac{\beta}{p-1}}
$$

if $d(x) \ll \lambda^{-\frac{1}{p-1}}$ as $\lambda \rightarrow \infty$.

As for the case B) corresponding to $q>\frac{r+1}{p^{\prime}}$, the critical scale becomes now (compare with Theorem 3, B)) $\lambda^{-\theta}$ with

and in fact the relevant estimate is,

$$
\theta=\frac{1}{p} \frac{r-p+1}{r-q+1}
$$

$$
\frac{A_{p}-\varepsilon}{\left(d(x)+A_{p}^{\frac{1}{\beta}} \lambda^{-\theta}\right)^{\beta}}-M \leq u_{\lambda} \leq \frac{A_{p}+\varepsilon}{\left(d(x)+A_{p}^{\frac{1}{\beta}} \lambda^{-\theta}\right)^{\beta}}+M,
$$

where $\varepsilon>0$ is prefixed and $d(x)<\delta, M=M(\varepsilon)$ and $\lambda \geq \lambda(\varepsilon)$. While the inner behavior (3.30) holds once again if $1 \gg d(x) \gg \lambda^{-\theta}$ as $\lambda \rightarrow \infty$, we find that

$$
u_{\lambda} \sim \lambda^{\frac{1}{q-p+1}},
$$

if $\lambda \rightarrow \infty$ and $x$ moves with $\lambda$ according to the scale $d(x) \ll \lambda^{-\theta}$.

Remark 1 . As in the linear diffusion problem, an intermediate layer behavior of the solution $u_{\lambda}$ to problem (1.13) is found when observing such solution in a multiple of the critical scale. For instance, in the case of problem (D),

$$
u_{\lambda} \sim \frac{A_{p}}{\left(1+\rho_{0}^{-1} A_{p}^{\frac{1}{\beta}}\right)^{\beta}} d(x)^{-\beta} \sim \frac{A_{p}}{\left(\rho_{0}+A_{p}^{\frac{1}{\beta}}\right)^{\beta}} \lambda,
$$

when $d(x) \ll 1$ according to $d(x) \sim \rho_{0} \lambda^{-\frac{1}{\beta}}$. Of course, similar estimates are obtained for the other boundary conditions by employing their own critical scales.

Acknowledgements. Supported by Ministerio de Ciencia e Innovacion and FEDER under grant MTM2008-05824 (Spain) and ANPCyT PICT 03-05009 and UBA X066 (Argentina). J. D. Rossi is a member of CONICET.

\section{REFERENCES}

[1] C. Bandle, M. Marcus, 'Large' solutions of semilinear elliptic equations: Existence, uniqueness and asymptotic behaviour, J. Anal. Math. 58 (1992), 9-24.

[2] M. Del Pino, R. Letelier, The influence of domain geometry in boundary blow-up elliptic problems, Nonlinear Anal. 48 (6) (2002), 897-904.

[3] E. DiBenedetto, $C^{1+\alpha}$ local regularity of weak solutions of degenerate elliptic equations, Nonlinear Anal. 7 (1983), no. 8, 827-850. 
[4] G. DíAz, R. Letelier, Explosive solutions of quasilinear elliptic equations: Existence and uniqueness, Nonlinear Anal. 20 (1993), 97-125.

[5] J. García-Melián, R. Letelier-Albornoz, J. SABina De Lis, Uniqueness and asymptotic behaviour for solutions of semilinear problems with boundary blow-up, Proc. Amer. Math. Soc. 129 (12) (2001), 3593-3602.

[6] J. García-Melián, J. Rossi, J. SABina DE Lis, A bifurcation problem governed by the boundary condition I. Nonlinear Differential Equations and Applications, NoDEA 14 (2007), 499-525.

[7] J. García-Melián, J. Rossi, J. Sabina De Lis, A bifurcation problem governed by the boundary condition II. Proc. London Math. Soc. 94 (2007), 1-25.

[8] J. García-Melián, J. Rossi, J. Sabina de Lis, Existence and uniqueness of positive solutions to elliptic problems with sublinear mixed boundary conditions. To appear in Comm. Contemp. Math.

[9] J. García-Melián, J. Rossi, J. SABina DE Lis, An elliptic system with bifurcation parameters on the boundary condition. J. Differential Equations 247 (2009), 779-810.

[10] J. B. Keller, On solutions of $\Delta u=f(u)$, Comm. Pure Appl. Math. 10 (1957), 503-510.

[11] G. Lieberman, Boundary regularity for solutions of degenerate elliptic equations, Nonlinear Anal. 12 (1988), 1203-1219.

[12] P. Lindqvist, On the equation div $\left(|\nabla u|^{p-2} \nabla u\right)+\lambda|u|^{p-2} u=0$, Proceedings of the Anerican Mathematical Society 109 (1990), 157-164.

[13] J. Matero, Quasilinear elliptic equations with boundary blow-up, J. Anal. Math. 69 (1996), 229-247.

[14] P. Tolksdorf, Regularity for a more general class of quasi-linear elliptic equations, J. Differential Equations 51 (1984), 126-150.

[15] J. L. VÁzQUEz, A strong maximum principle for some quasilinear elliptic equations, Appl. Math. Optim. 12 (1984), no. 3, 191-202.

J. García-Melián and J. C. Sabina de Lis

Departamento de Análisis Matemático, Universidad de la laguna.

C/. Astrofísico Francisco SÁnchez s/n, 38271 - La Laguna, SPAIN

and

Instituto Universitario de Estudios Avanzados (IUdEA) en Física Atomica,

Molecular y Fotonica, Facultad de Física, Universidad de La Laguna

C/. Astrofísico Francisco SÁnchez s/n, 38203 - La Laguna, SPAIN

E-mail address: jjgarmel@ull.es, josabina@ull.es

J. D. Rossi

Departamento de Matemática, FCEyn UBA,

Ciudad Universitaria, Pab 1 (1428)

Buenos Aires, Argentina.

E-mail address: jrossi@dm.uba.ar 